\title{
LA-UR-21-24543
}

Approved for public release; distribution is unlimited.

Title:

Author(s):

Intended for:

Issued:

\section{CAREER PATH PROGRESSIONS PROJECT LANL Team}

Ramsay, Susan M.

Collaboration

2021-05-11 
Disclaimer:

Los Alamos National Laboratory, an affirmative action/equal opportunity employer, is operated by Triad National Security, LLC for the National Nuclear Security Administration of U.S. Department of Energy under contract 89233218CNA000001. By approving this article, the publisher recognizes that the U.S. Government retains nonexclusive, royalty-free license to publish or reproduce the published form of this contribution, or to allow others to do so, for U.S. Government purposes. Los Alamos National Laboratory requests that the publisher identify this article as work performed under the auspices of the U.S. Department of Energy. Los Alamos National Laboratory strongly supports academic freedom and a researcher's right to publish; as an institution, however, the Laboratory does not endorse the viewpoint of a publication or guarantee its technical correctness. 


\section{Los Alamos NATIONAL LABORATORY}

\section{CAREER PATH PROGRESSIONS PROJECT LANL Team}

Susan Ramsay, ORI-1

April 22, 2021

Replace and add LA-UR number 


\section{Introductions - LANL Project Sponsors}
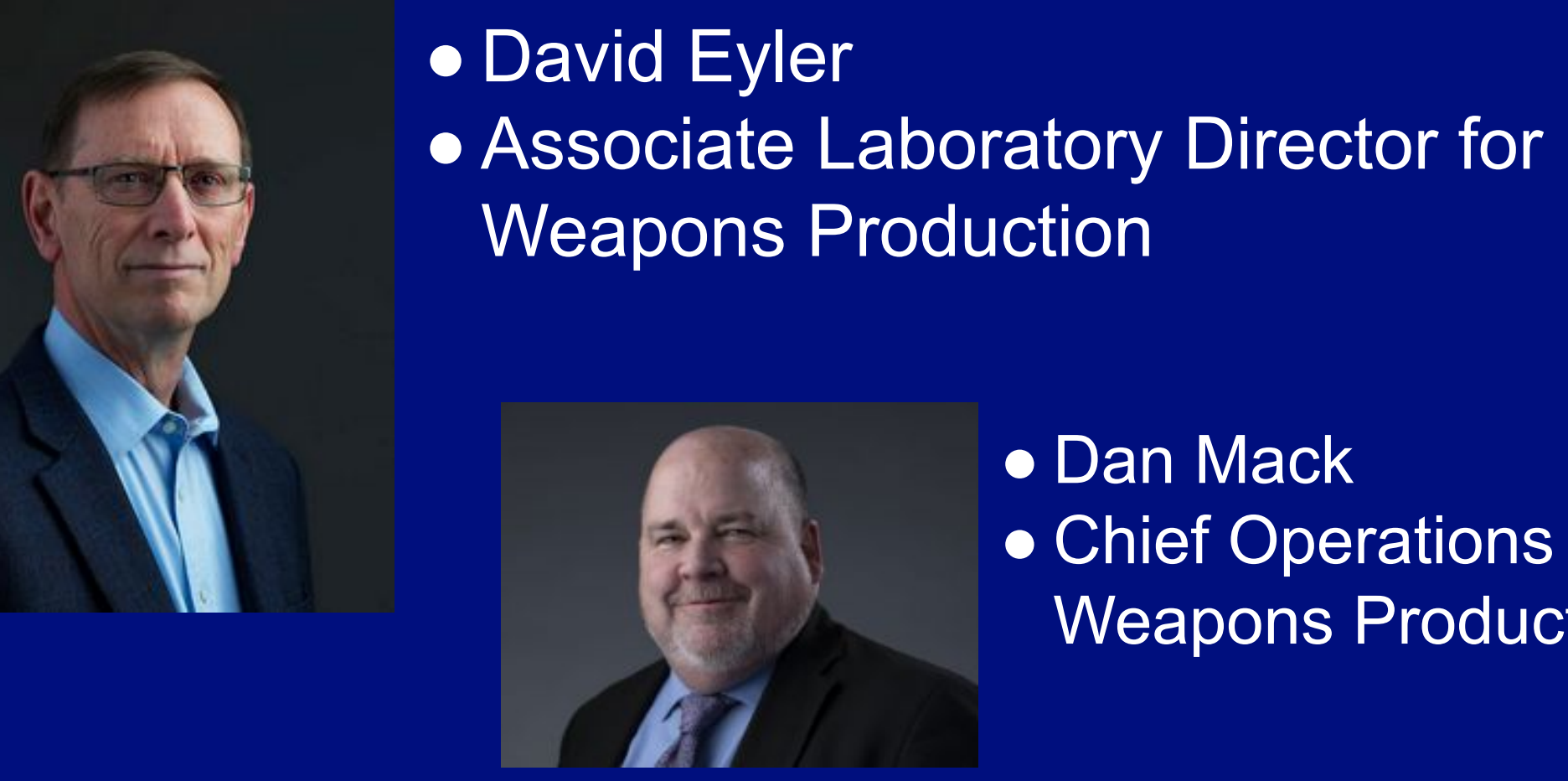

- Dan Mack

- Chief Operations Officer, Weapons Production 


\section{Introductions - LANL Strategic Leads}

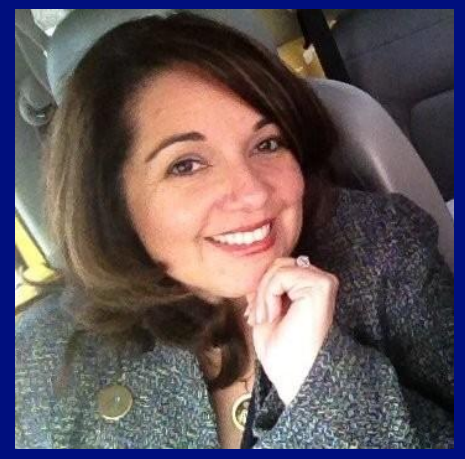

Jackie Valdez, Program Manager Human Capital Strategy for Weapons Production jvaldez@lanl.gov, 5056678385

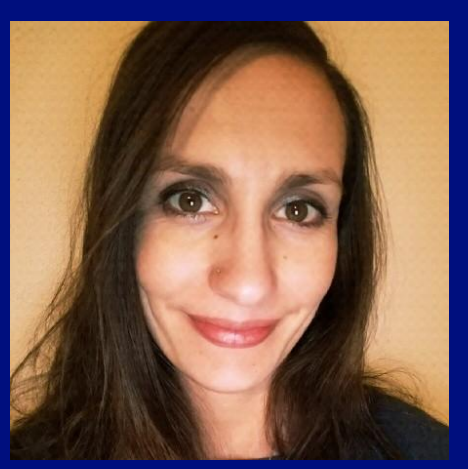

Evelena Valencia, Group Leader ORI-1 Training \& Mission Services evelena@lanl.gov, 5056657317 


\section{Introductions - LANL Core Project Team}

- Kirsty Archuleta, Staff Operations Manager, PT-DO Pit Technologies Division kirsty@lanl.gov, 5056670697

- Ryan Chavez, Team Lead-Technical Project Manager \& Subcontract Technical Representative ORI-1 Training \& Mission Services, ryancha@lanl.gov, 5056679993

- Brooke Jackson, Chair Employee Development Network, HR-POD HR People \& Organization Development bmiack@lanl.gov, 2083603392

- Mia Logan, Group Leader, HR-POD HR People \& Organization Development mlogan@lanl.gov

- Justin Najaka, Compensation Analyst, HR-CWDA Human Resources - Compensation irn@lanl.gov, 5053092541

- Susan Ramsay, Technical Project Manager, ORI-1 Training \& Mission Services ramsay@lanl.gov, 5056958274

- Robin Simpson, Staff Operations Manager, Weapons Production robin s@lanl.gov, 5056659459

- Jessica Vigil, Human Resources Generalist, HR-CFS Human Resources Central \& Field Services jessica m@lanl.gov, 5056955941 


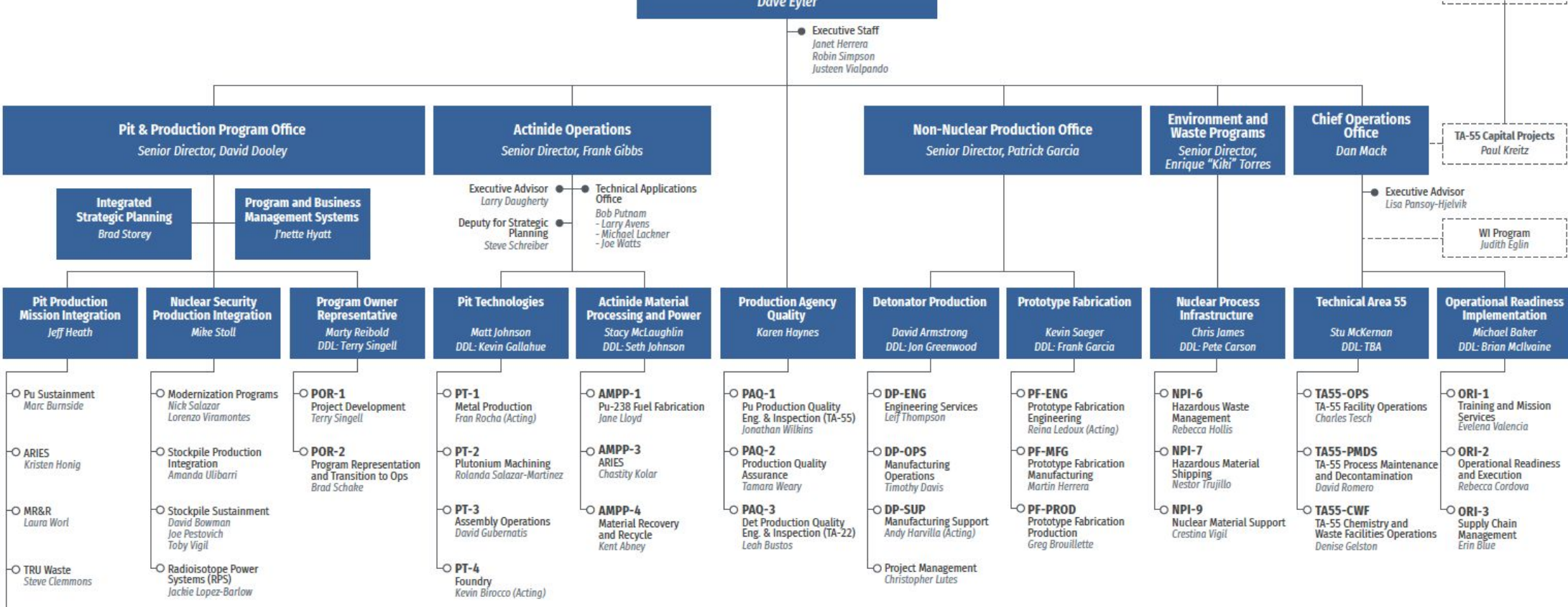

-0 Container Stroge
Cristy Abeyta

$\underset{\text { Leisa Davenhall }}{\mathrm{AmO}_{2}}$

- Subcrit Components
Leisa Davenhall

LO Office of Experimental Science
Anthony fredentourg 


\section{Key LANL Stakeholders}

- AMPP Actinide Material Processing \& Power Division

Stacy McLaughlin, Division Leader

J. Seth Johnson, Deputy Division Leader

- DP Detonator Production Division

David Armstrong, Division Leader

Jon Greenwood, Deputy Division Leader

- NPI Nuclear Process Infrastructure Division

Chris James, Division Leader

Peter Carson, Deputy Division Leader

- PF Prototype Fabrication Division

Kevin Saeger, Division Leader

Frank Garcia, Deputy Division Leader

- PT Pit Technologies Division

Matt Johnson, Division Leader

Kevin Gallahue, Deputy Division Leader 